\section{Sommer mit Risiken}

m Mai hat die Saison der Bienen und

Wespen begonnen. Jetzt können wieder gehäuft schwere allergische Reaktionen auf Insektengift auftreten. Ärzte sollten Patienten mit Insektengiftallergie und bekanntem Risiko für anaphylaktische Reaktionen in dieser Zeit darauf hinweisen, Insektenkontakte möglichst $\mathrm{zu}$ vermeiden und sich besonders umsichtig $\mathrm{zu}$ verhalten. In vielen Fällen ist auch die Verordnung von Notfallmedikamenten ratsam, denn anaphylaktische Reaktionen infolge eines Bienen- oder Wespenstichs können einen sehr schnellen und lebensbedrohlichen Verlauf nehmen. Ein optimal ausgestattetes Notfallset sollte einen Adrenalin-Autoinjektor (z. B. Fastjekt ${ }^{\circledR}$ oder Fastjekt ${ }^{\circledR}$ Junior für Kinder), ein H1-Antihistami-

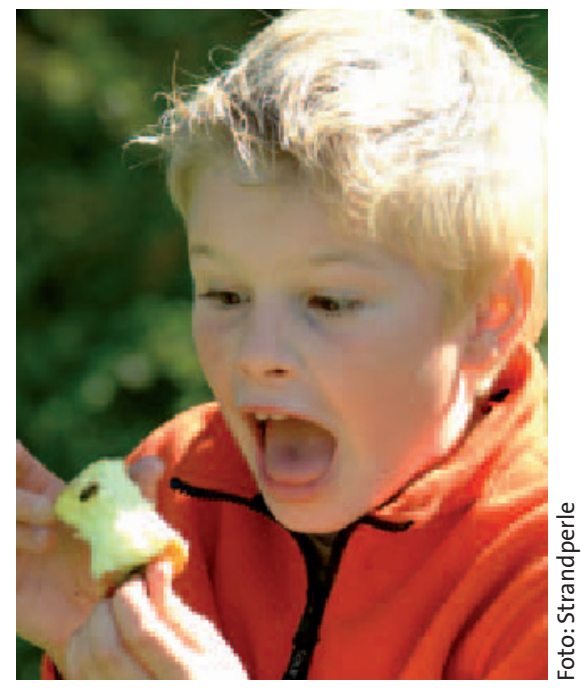

Zum Glück hat er die Wespe bemerkt.

nikum und ein Glukokortikoid enthalten. Den Einsatz eines Adrenalin-Auto- injektors können Ärzte mit ihren Patienten gemeinsam einüben, beispielsweise mit einem speziellen Trainer, den Ärzte per Fax unter 0 40/72 765268 direkt bei Allergopharma kostenlos bestellen können. Wichtig ist auch, die Patienten daran zu erinnern, bei einer anaphylaktischen Reaktion nach der Medikamentengabe immer den Notarzt zu alarmieren. Außerdem sollten Insektengiftallergiker noch vor dem ersten Flug der Insekten die Haltbarkeit ihrer Notfallmedikamente überprüfen und das Notfallset immer zur Hand haben. Gerade wenn die akute Gefahr nur zu einer bestimmten Zeit im Jahr besteht, vergessen dies viele Patienten. Einen speziellen Erinnerungsservice bietet die Website www. fastjekt.de.

Nach Informationen von Allergopharma, Reinbek

\title{
Flexibles Therapieschema bei Asthma
}

$\mathrm{D}$ ie neuen GINA (Global Initiative for Asthma)-Empfehlungen stellen die Asthmakontrolle in den Mittelpunkt der Therapie und nicht mehr den Schweregrad der Erkrankung wie bisher, erläuterte Prof. Dr. Roland Buhl, Mainz, auf einem Symposium im Rahmen des DGIM-Kongresses in Wiesbaden. Die Asthmakontrolle kann dabei eine fortwährende Anpassung der Behandlung an die veränderten Gegebenheiten der Er- krankung bedeuten. Einfluss haben die Symptomatik, funktionelle Einschränkungen, Exazerbationen und eine modifizierte Bedarfs- und Notfallmedikation. Die Behandlung wird entsprechend dem aktuellen Status des Patienten eskaliert oder deeskaliert. Als Bedarfssubstanzen stehen weiterhin kurzwirksame Beta-2Sympathomimetika zur Verfügung. Als Basismedikation des Asthma werden nach wie vor inhalative Kortikosteroide

\begin{tabular}{|c|c|c|c|}
\hline Parameter & Kontrolliert & Teilweise kontrolliert & Unkontrolliert \\
\hline Symptome am Tag & $\begin{array}{l}\text { keine } \\
(\leq 2 \times / \text { Woche })\end{array}$ & öfter als $2 \times /$ Woche & \\
\hline $\begin{array}{l}\text { Einschränkung der } \\
\text { Aktivitäten }\end{array}$ & keine & jede & 3 oder mehr \\
\hline $\begin{array}{l}\text { Nächtliche Symptome/ } \\
\text { Erwachen }\end{array}$ & keine(s) & jede & $\begin{array}{l}\text { Kriterien eines } \\
\text { teilweise kontrol- }\end{array}$ \\
\hline $\begin{array}{l}\text { Bedarfs-/ } \\
\text { Notfallmedikation }\end{array}$ & $\begin{array}{l}\text { keine } \\
(\leq 2 \times / \text { Woche })\end{array}$ & öfter als $2 \times /$ Woche & $\begin{array}{l}\text { lierten Asthmas } \\
\text { erfüllt }\end{array}$ \\
\hline $\begin{array}{l}\text { Lungenfunktion } \\
\text { (PEF oder FEV } 1 \text { ) }\end{array}$ & normal & $\begin{array}{l}<80 \% \text { (Soll oder } \\
\text { persönlicher Bestwert) }\end{array}$ & \\
\hline Exazerbationen & keine & eine oder mehrere/Jahr* & $\begin{array}{l}\text { eine in beliebige } \\
\text { Woche }^{\#}\end{array}$ \\
\hline
\end{tabular}

(ICS) angesehen. Ist ein Asthma mit einer niedrigen ICS-Dosis allein nicht $\mathrm{zu}$ kontrollieren, ist die zusätzliche Gabe eines langwirksamen Beta-2-Mimetikums (LABA) in freier oder fester Kombination die am ehesten Erfolg versprechende Option, wie Buhl berichtete. „Wichtig ist, dass eine regelmäßige Therapie mit LABA in der Dauertherapie nur in freier oder fester Kombination mit einem ICS angewendet werden sollte." Die Beclometason-Formoterol-Fixkombination (Inuvair ${ }^{\circledR}$ Mikrosol) war in einer Studie [Bonnet Gonod et al. ERJ 2006; 28: 205s] der vom IQWIG vorgeschlagenen Einzelapplikation des Steroids und des LABA in vielen für die Asthmakontrolle relevanten Punkten - etwa den Tagen ohne Symptome - signifikant überlegen. Buhl wies dabei ausdrücklich darauf hin, dass aufgrund der Studienbedingungen die Vorteile der alltäglichen Compliance bei einer Fixkombination noch gar nicht in den Daten enthalten waren.

Symposium „Atemwegserkrankungen: Neue Konzepte - neue Rezepte“. 114. Kongresses der Deutschen Gesellschaft für Innere Medizin Wiesbaden, 29. März bis 2. April 2008 Veranstalter: Janssen-Cilag, Neuss 